\title{
Parametrização de Campo de Força e Estudo Teórico do Comportamento Estrutural e Eletrônico de FAD e TPP - Cofatores da Enzima AHAS.
}

\author{
Renan F. Guerra \& Eduardo F. Franca
}

\section{Introdução}

A enzima Acetohidroxiácido Sintase (AHAS) é encontrada em plantas e microrganismos já que está envolvida nos processos catalíticos e de biossíntese dos aminoácidos de cadeia ramificada (ACR). A AHAS é a primeira da rota biossintética dos mesmos e, portanto, alvo de herbicidas inibitórios que são usualmente empregados na agricultura, as sulfonilureias e imidazolinonas ${ }^{1}$. Dessa forma, pode ser utilizada como alvo biológico, em métodos analíticos baseados em Microscopia de Força Atômica, na deteç̧ão desses agrotóxicos para controle ambiental mais rigoroso de culturas agrícolas para exportação.

A AHAS para exercer sua atividade catalítica necessita fundamentalmente de cofatores como os íons $\mathrm{Mg}^{2+}$ e $\mathrm{K}^{+}$, e as biomoléculas FAD (Dinucleotideo de Flavina e Adenina) e TPP (Tiamina Difosfato), como mostrado na Figura 1a e 1b, respectivamente. O papel dessas biomoléculas na enzima ainda permanece um enigma, mas sabe-se que são fundamentais para a integridade estrutural e atividade enzimática da mesma $^{2}$. a

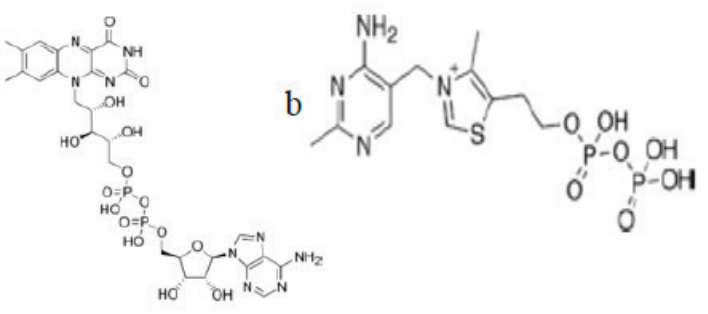

Figura 1. Estrutura molecular dos cofatores a) FAD e b) TPP.

O presente trabalho objetivou estudar o efeito da água na conformação estrutural e nas propriedades eletrônicas das moléculas de FAD e TPP em solução aquosa, haja vista que as propriedades moleculares desses co-fatores é afetada significativamente em fase condensada. Para tanto, foi utilizado um método híbrido de mecânica quântica e mecânica molecular (clássica), conhecido como QM/MM Sequencial, considerado adequado para estudos com esse foco já que a região de interesse (biomolécula) é tratada quanticamente e o meio no qual se encontra inserida (solvente) é tratada por cálculos 
clássicos demandando menor custo computacional que os métodos puramente quânticos.

\section{Métodos}

Previamente, o campo de força das moléculas de FAD e TPP foram obtidos online utilizando o programa SwissParam ${ }^{3}$. Depois disso, as moléculas de FAD (carga -1) e TPP (carga -2) foram colocadas separadamente em uma caixa de simulação retangular a 1,5 nm do sistema e solvatada com moléculas de água do tipo Simple Point Charge (SPC) mantido em esemble NPT (298 K and 1 bar). Íons sódio foram adicionados para neutralizar a carga do sistema. As dinâmicas moleculares foram realizadas utilizando o programa GROMACS 4.6 e o campo de força CHARMM 2.7 durante 4 e 10 ns para FAD e TPP, respectivamente.

Para cada ps de simulação a estrutura eletrônica das moléculas foi obtida utilizando cálculos semi-empíricos single point, com o halmitoniano PM7 e o programa MOPAC 2012.

Os resultados foram analisados utilizando os programas JMol, VMD e GRACE.

\section{Resultados e Discussão}

Para a estrutura do FAD o programa SwissParam gerou todos os parâmetros de campo de força (ligados e nãoligados) adequados para a descrição do composto utilizando simulação por dinâmica molecular. Para a molécula do TPP nem todos os diedros próprios foram identificados pela ferramenta online e, portanto, não tiveram seus parâmetros gerados. Na Tab. 1 estão listados os diedros não identificados para a estrutura do TPP (a cada linha da tabela os parâmetros para os diedros são os mesmos).

Tabela 1. Diedros próprios não identificados pelo programa SwissParam para a molécula TPP e parametrizados separadamente.

\begin{tabular}{|c|c|c|c|c|}
\hline \multirow{3}{*}{1} & & H1 & \multirow{3}{*}{$\mathrm{N} 1 \mathrm{C} 2 \mathrm{C} 1$} & H1 \\
\hline & $\mathrm{C} 3 \mathrm{C} 2 \mathrm{C} 1$ & $\mathrm{H} 2$ & & $\mathrm{H} 2$ \\
\hline & & H3 & & H3 \\
\hline \multirow{3}{*}{2} & & $\mathrm{C} 12$ & \multirow{3}{*}{$\mathrm{C} 2 \mathrm{C} 3 \mathrm{~S} 1$} & $\mathrm{C} 12$ \\
\hline & $\mathrm{C} 2 \mathrm{C} 3$ & H13 & & H13 \\
\hline & C11 & $\mathrm{H} 14$ & & $\mathrm{H} 14$ \\
\hline \multirow{3}{*}{3} & & C6 & \multirow{3}{*}{$\mathrm{N} 1 \mathrm{C} 5 \mathrm{C} 4$} & C6 \\
\hline & $\mathrm{N} 1 \mathrm{C} 5 \mathrm{C} 2$ & $\mathrm{H} 5$ & & H5 \\
\hline & & H6 & & H6 \\
\hline
\end{tabular}

Dessa forma, a alternativa encontrada foi obter tais parâmetros a partir de uma molécula com estrutura análoga à do TPP. Gutowski (1979) propôs estruturas análogas ao Thiamine Pyrophosphate (TPP) de modo que a estrutura mais semelhante é o Thiamine Thiothiazolone Pyrophosphate (TTTPP) - a única diferença entre as estruturas é o átomo representado em rosa (H para o TPP e S para o TTTPP) conforme apresentado na Fig. 2 onde está apresentada a estrutura genérica para o TPP e seu análogo5.

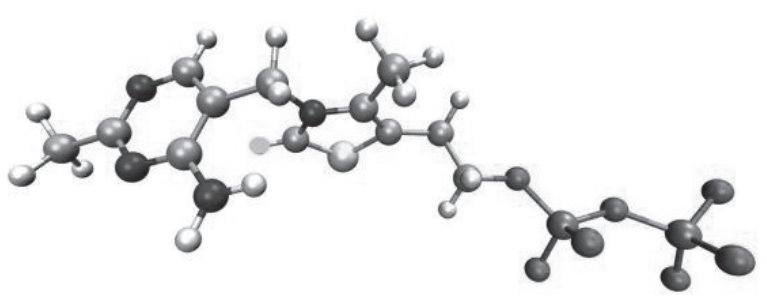

Figura 2. Estrutura geral do TPP e seus análogos.

Para a estrutura do TTTPP os diedros foram adequadamente descritos e os parâmetros utilizados na descrição do TPP no campo de força. Após a parametrização foi aplicada a metodologia QM/MM para ambos cofatores da enzima AHAS.

O cálculo da raiz quadrada do desvio quadrático médio (RMSD) com relação à estrutura inicial aponta para um desvio máximo de 0,46 e $0,34 \mathrm{~nm}$ para as moléculas de FAD e TPP, respectivamente, relacionado a flutuações conformacionais das estruturas em solução aquosa ao longo da trajetória. Isso está relacionado ao fato de a molécula de FAD apresentar uma estrutura consideravelmente maior e, portanto, maior número de graus de liberdade.

Em relação aos orbitais de fronteira, $\mathrm{HOMO} \mathrm{e}$ LUMO, verificou-se que a variação estrutural para a molécula do FAD resultou na diminuição de energia em aproximadamente 0,295 eV. Os orbitais HOMO encontram-se localizados no agrupamento $\mathrm{SO}_{4}$ tanto para estrutura inicial quanto para a estrutura inicial (Fig. 3a). Para a estrutura mais estável (Fig. 3b), no entanto, ocorre um deslocamento do HOMO diminuindo a extensão do mesmo de 5 para apenas 2 átomos, conforme apresentado nas representações das Fig. 3a e $3 b$. 
a

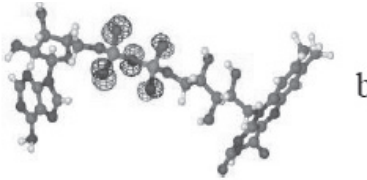

b
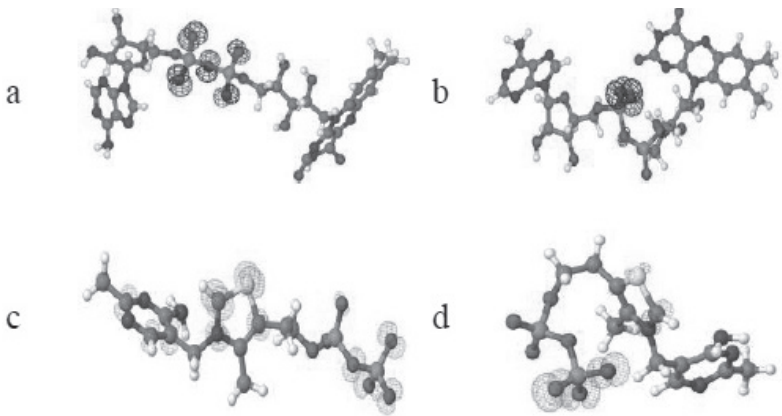

Figura 3. Orbitais moleculares HOMO nas estruturas inicial (a e c) e mais estável em solução aquosa (b e d) para as moléculas de FAD e TPP, respectivamente.

Para a molécula de TPP a estrutura mais estável ocorre após aproximadamente $7 \mathrm{~ns}$ de simulação, de modo que a diminuição energética ocasionada pela variação estrutural é de $0,574 \mathrm{eV}$. Esta estabilização energética se deve ao fato da relevante variação na conformação estrutural sofrida pela molécula, conforme se observa ao comparar as estruturas apresentadas nas Fig. $3 \mathrm{c}$ e $3 \mathrm{~d}$. Os orbitais HOMO na estrutura inicial se encontram distribuídos ao longo da molécula, no entanto, na estrutura mais estável os mesmos se encontram predominantemente localizados sob os átomos de oxigênio do agrupamento $\mathrm{PO}_{4}$ da extremidade da estrutura.

A estrutura mais estável da molécula do FAD se apresenta numa conformação mais fechada ocasionada pela ocorrência de duas ligações de hidrogênio intramoleculares (Fig. 4) que apresentam tempo de vida médio de 3,49 ps e momento dipolar em solução aquosa maior em 1,97 Debyes. No entanto, para a molécula do TPP a considerável variação na estrutura é gerada apenas pelo calor de formação que é de cerca de - 166,97 kJ.mol ${ }^{-1}$.

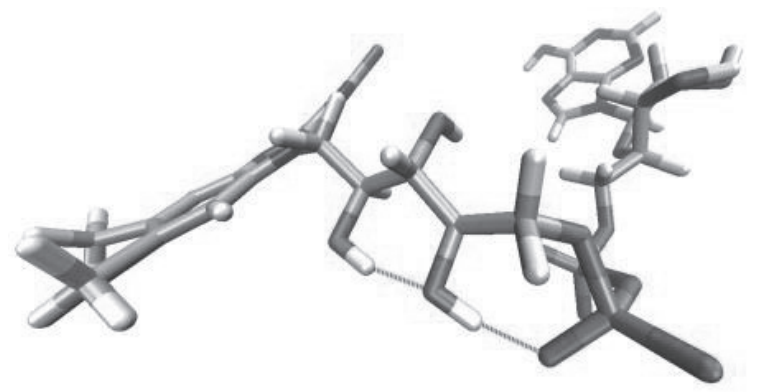

Figura 4. Ligações de Hidrogênio (vermelho) responsáveis pelo conformação mais estável da estrutura.
Dessa forma, torna-se possível afirmar que as moléculas de água tratadas como cargas pontuais foram responsáveis pela amostragem de estruturas mais estáveis para as duas moléculas em estudo.

\section{Conclusões}

O programa SwissParam foi eficiente e útil no processo de parametrização das moléculas do FAD e TPP, promovendo uma economia de tempo e memória computacional nessa etapa essencial das metodologias computacionais clássicas. A metodologia de QM/MM sequencial permitiu a descrição adequada do efeito do solvente explícito, temperatura e pressão na estrutura eletrônica do FAD e do TPP reafirmando a validade dos métodos híbridos no estudo de propriedades termodinâmicas de moléculas com elevado grau de liberdade como as estudadas.

Estruturas mais estáveis foram obtidas pela influência do solvente na estrutura eletrônica das moléculas de modo que as informações do presente trabalho serve de base para o estudo mais detalhado da função desempenhada pelos cofatores na enzima AHAS.

\section{Agradecimentos}

Os autores agradecem o apoio concedido pela CAPES, CNPQ, FAPEMIG e RQ-MG.

\section{References}

1. J. A. MCourt. R. G. Duggleby. Amino Acids. 31 (2), 173- 10,(2006)

2. J. A. MCourt. et. al. Proc. Natl. Acad. Sci. 103 (3), 569-573,(2006).

3. V. Zoete, M. A. Cuendet, A. Grosdidier, O. Micheelin. J. Comput. Chem. 32(11), 2359-2368, (2011).

4. B. R. BROOKS. et al. J. Comput. Chem., 30 (10), 15451614,(2009).

5. J. A. Gutowski, G. E. Lienhard. The Journal of Biological Chemistry. 251 (9), 2863-2866,(1976).

\section{Renan F. Guerra* \& Eduardo de Faria Franca}

a Laboratório de Cristalografia e Química Computacional - Instituto de Química da Universidade Federal de Uberlândia (UFU)

*E-mail: renan_s13@hotmail.com 